\title{
Combined Heart-Kidney Transplantation: A Single Center Experience
}

\author{
Giuliana Bacchi ${ }^{1}$, Luciano Potena ${ }^{2}$, Giovanni Mosconi ${ }^{1}$, Candida C. Quarta ${ }^{2}$, Lucia Stalteri ${ }^{1}$, \\ Gaia Magnani $^{2}$, Giorgio Feliciangeli ${ }^{1}$, Francesco Grigioni $^{2}$, Angelo Branzi ${ }^{2}$, Sergio Stefoni ${ }^{1}$ \\ ${ }^{1}$ Department of Internal Medicine, Aging and Renal Disease, University of Bologna, Bologna, Italy \\ ${ }^{2}$ Cardiovascular Department, heart transplant program, University of Bologna, Bologna, Italy \\ E-mail: giuliana.bacchi@unibo.it \\ Received August 26, 2011; revised October 1, 2011; accepted October 12, 2011
}

\begin{abstract}
Introduction: We report our experience with seven cases of combined heart-kidney transplantation (HKT). Patients and methods: Between January 2003 and December 2009, seven subjects underwent combined HKT, receiving both organs from a single donor. Their age ranged from 30 years to 59 years, six were male. Five patients were dialysis dependent before transplantation and two were in chronic renal failure (serum creatinine levels $>2.6 \mathrm{mg} / \mathrm{dL}$ ). The heart was transplanted first in all cases. Results: Heart function rapidly re-covered in five of the patients, while two needed temporary inotropic and mechanical support. Diuresis started immediately in four patients. At discharge, all patients had well-functioning grafts (left ventricular ejection fraction $60 \% \pm 6 \%$; serum creatinine $1.4 \pm 0.3 \mathrm{mg} / \mathrm{dL}$ ). After an average follow-up period of $45 \pm 24$ months no deaths have occurred. Heart allografts are functioning normally in six patients and none of the patients currently require dialysis treatment. The main adverse event noted during follow-up was hypertension in five patients. Four patients were cardiac allograft rejection free and five patients were kidney rejection free. Conclusion: Our results are in line with the data which has been previously reported in the literature and suggest that HKT is a viable therapeutic choice in the treatment of advanced cardiac and renal failure in carefully selected patients.
\end{abstract}

Keywords: Heart Transplantation, Kidney Transplantation, Dual-Organ Transplantation, Heart Failure, Kidney Failure

\section{Introduction}

Since the first report of a combined heart and kidney transplant (HKT) from a single donor in 1978 [1], and following the subsequent successful HKT in terms of long term survival in 1986 [2], this therapeutic solution has been performed numerous times and with growing frequency with more than 722 such procedures reported to the United Network for Organ Sharing (UNOS) between January 1, 1988 and May 31, 2011.

Herein, we report our experience with 7 patients who underwent combined HKT.

\section{Methods}

Between January 2003 and December 2009, 533 kidney transplantations and 243 heart transplantations were performed at the S. Orsola University Hospital. Among these, seven subjects underwent combined heart and kidney transplantations (HKT), receiving both organs from single donors selected according to ABO blood group compatibility and a negative lymphocytotoxic cross-match, but without regard to HLA-antigen matching due to the time constraints of cardiac surgery. One other patient, in addition to a heart and kidney, received a liver and so was not considered in this report. A retrospective review of the medical records of these 7 HKT recipients was conducted.

The surgical technique did not differ from the one used in the isolated transplantation of these organs.

In six cases, the heart transplant was performed and then the renal allograft was positioned immediately afterwards. In one case (no. 3), the kidney was implanted ten hours after the heart when the hemodynamic situation of the patient had stabilized.

Patients were followed up by both transplantation teams, 
and surveillance did not differ from that of recipients of isolated organs.

Coronary angiography and echocardiography examinations were performed at regular intervals to assess heart function. For the surveillance of cardiac allograft rejection, endomyocardial biopsy was routinely performed once a week during the first month, bimonthly for the next 2 months, monthly for the next 3 months, every two months during the six to twelve month period after transplantation, quarterly for the second year and twice a year thereafter. Acute heart rejection was histologically graded according to the international society of heart and lung transplantation classification (ISHLT).

For the kidney, function was generally checked monthly by means of blood and urine tests; duplex Doppler ultrasonography was performed every 4 months - 6 months. Renal rejection was suspected upon biochemical parameters and by means of the Doppler ultrasonography resistance index perfusion scan technique. Renal biopsy was not performed routinely but only in the case of a persistent compromise of renal function. Biopsy was performed in one patient (no. 7).

Immunosuppression: all patients were treated according to the protocols for immunosuppressive therapy in heart transplant recipients, consisting in induction with rabbit anti-thymocyte globulin (rATG), (Thymoglobuline, Genzyme; Ireland), followed by maintenance therapy with cyclosporine, steroids and azathioprine (no.1 - no.3) or mycophenolate mofetil (no. 4 - no. 7). Rejection was treated with pulse intravenuous steroids or with intravenous rATG if hemodynamic compromise was present.

\section{Results}

Patient characteristics are summarized in Table 1. Patient age ranged from 30 years to 59 years with a mean of 40.5 years \pm 10.3 years, all of the recipients were male except for one female. Five patients had a normal body mass index, one was overweight and the female was underweight. The etiology of the end-stage heart failure was idiopathic cardiomyopathy in four patients, hypertrophic cardiomyopathy in two, and ischemic cardiomyopathy in one. This latter patient had previously undergone valvular cardiac surgery. Each patient had severe, fixed impairment of systolic cardiac function with an average left ventricle ejection of $27 \% \pm 10 \%$.

Five patients had irreversible end-stage renal failure managed by dialysis with a mean time on dialysis of 55 months \pm 63 months. The other two were suffering from chronic renal failure with serum creatinine levels $>2.6$ $\mathrm{mg} / \mathrm{dL}$.

Donor and allograft ischemic times are shown in Table 2.

Six of the donors were male and were aged between 17 and 60 (mean age 30 years \pm 18 years). The mean ischemic time was 160 minutes \pm 14 minutes for the

Table 1. Patient demographics, clinical characteristics at the time of transplantation.

\begin{tabular}{|c|c|c|c|c|c|c|c|c|c|}
\hline Pt no. & Age & Sex & Body Mass Index & Heart disease & LVEF (\%) & $\begin{array}{l}\text { Cardiax index } \\
\left(\mathrm{L} / \mathrm{min} / \mathrm{m}^{2}\right)\end{array}$ & Kidney disease & Dialysis type & $\begin{array}{c}\text { Time on } \\
\text { Dialysis months }\end{array}$ \\
\hline 1 & 59 & M & 26 & ICM & 23 & 2.23 & VA & $2.6^{*}$ & - \\
\hline 2 & 32 & M & 23 & IDCM & 20 & 1.79 & GN & HD & 1 \\
\hline 3 & 49 & M & 22 & HCM & 29 & 1.91 & IN & PD & 11 \\
\hline 4 & 38 & $\mathrm{~F}$ & 17 & HCM & 48 & 3.31 & IN & HD & 23 \\
\hline 5 & 34 & M & 20 & IDCM & 28 & 2.92 & VA & $3.1^{*}$ & - \\
\hline 6 & 30 & M & 25 & IDCM & 20 & 4.58 & GN & HD & 92 \\
\hline 7 & 42 & M & 20 & IDCM & 21 & 1.16 & ADPKD & HD & 148 \\
\hline
\end{tabular}

*Serum creatinine levels. ADPKD, autosomal dominant polycystic kidney disease; GN, chronic glomerulare disease; HCM, hypertrophic cardiomyopathy; HD, hemodialysis; ICM, ischemic cardiomyopathy; IDCM idiopathic dilatative cardiomyopathy; IN, chronic interstizial nephritis; LVEF left ventricular ejection fraction; PD, peritoneal dialysis

Table 2. Donors and allograft ischemic time.

\begin{tabular}{|c|c|c|c|c|c|}
\hline Pt no. & Donor age & Donor sex & Heart ischemic time minutes & Kidney ischemic time hours & Cross-match \\
\hline 1 & 18 & $\mathrm{M}$ & 180 & 10 & negative \\
\hline 2 & 16 & M & 170 & 10 & negative \\
\hline 3 & 31 & M & 145 & 20 & negative \\
\hline 4 & 60 & $\mathrm{~F}$ & 170 & 11 & negative \\
\hline 5 & 17 & M & 144 & 9 & negative \\
\hline 6 & 20 & $\mathrm{M}$ & 164 & 10 & negative \\
\hline 7 & 49 & $\mathrm{~F}$ & 148 & 8 & negative \\
\hline
\end{tabular}


cardiac allograft and 11 hours \pm 4 hours for the kidney allograft. The clinical results and outcomes are summarized in Table 3.

Heart function recovered rapidly after transplant in five patients who only needed low doses of inotropic drugs. Two patients (no.3 and no. 6), presented with delayed recovery of heart function which required increased inotropic support and (no. 6) intra-aortic balloon pump assistance (IABP). Nevertheless, in both cases the kidney was successfully transplanted with a 10 hour delay in patient no.3 and immediately after the implant of the IABP in patient no 6. Diuresis was immediate in four patients and delayed in the other three (no.1, no.3 and no. 7) who required haemodialysis for 16, 3 and 4 days, respectively.

The duration of postoperative hospitalization ranged from 25 days to 58 days and at discharge time, both grafts in all patients were well-functioning (mean left ventricular ejection fraction $60 \% \pm 6.2 \%$; mean serum creatinine $1.4 \mathrm{mg} / \mathrm{dL} \pm 0.3 \mathrm{mg} / \mathrm{dL}$ ). During the follow-up period, one patient (no. 2) was free of adverse events, while five patients presented with hypertension which is being treated with anti-hypertensive drugs. Insulindependent diabetes mellitus developed in patient no. 1 and patient no. 4 , while patient no. 7 experienced a urinary tract infection. Chronic obstructive pulmonary disease was noted in patient. no. 1 .

As regards organ rejection, 3 patients experienced cardiac allograft rejection (43\%) and 2 patients experienced kidney rejection (28\%). There were a total of seven cardiac allograft rejection episodes, clinically significant in three patients (grade 3A, ISHLT ) and of these three patients, two also experienced a kidney rejection episode.

In the two patients that experienced both kidney and heart rejection, these episodes were independent of each other.

After a mean follow-up period of 44.8 months \pm 24.4 months, all the patients are still alive.

Six of the cardiac allografts still have normal systolic function and are New York Heart Association (NYHA) class I or II. The worst current cardiac function (EF 42\%) is that of patient no. 1 , who is also the oldest of the patients and has the longest graft survival time in our series, with 75 months of follow-up. Unfortunately, 58 months after HTX he developed emphysema and is now NYHA class III.

All of the kidney allografts continue to function satisfactorily with a mean serum creatinine of $1.2 \mathrm{mg} / \mathrm{dL} \pm$ $0.2 \mathrm{mg} / \mathrm{dL}$ in six patients, while in pt no. 7, an increase in serum creatinine concentration $(2.9 \mathrm{mg} / \mathrm{dL})$ has been noted.

In fact, patient no. 7 already had impaired renal function at the second month following recurring episodes of sepsis (Escherichia coli) originating from his native polycystic kidney. Three months after HKT, he underwent left nephrectomy, it was not possible to perform the operation before this time due to the severely compromised cardiac status of the patient. Biopsy was performed 6 months after HKT and revealed signs of renal tubulophathy. No functional improvement was noticed after the reduction of the calcineurin inhibitors.

\section{Discussion}

Several studies regarding combined heart-kidney transplantation supports this option as a promising opportunity for patients with the co-existing failure of both organs.

Although one of the first multi-institutional studies [3] of 82 HKT reported a survival rate 2-year lower than

Table 3. Patient outcome data.

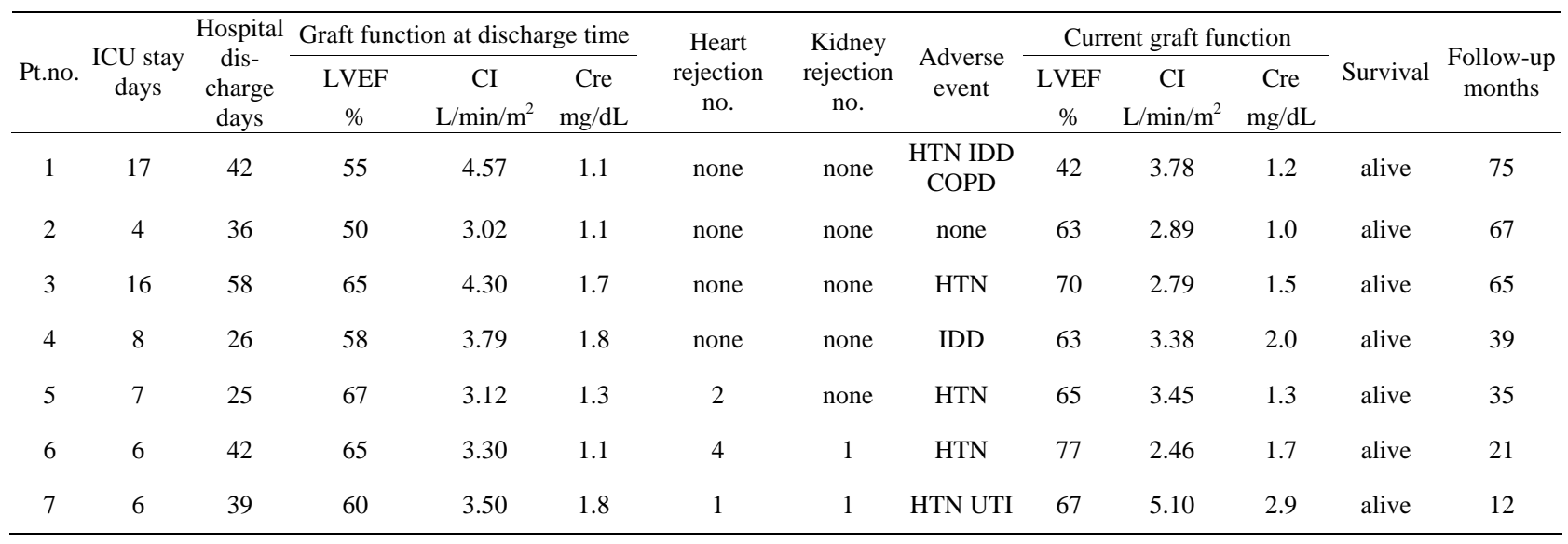

CI, cardiac index; COPD, chronic obstructive pulmonary disease; Cre, serum creatinine; HTN, hypertension; ICU, intensive care unit; IDD, insulin-dependent diabetes; LVEF left ventricular ejection fraction; UTI, urinary tract infection. 
that of isolated heart transplant recipients, according to the UNOS database, this difference was not statistically significant; several more recent studies from single centres with a low number of cases have yielded encouraging results, both in terms of long-term graft function and patient survival [4-7].

Moreover, in a recent analysis Russo et al [8], referring also to the UNOS database, found that low risk patients with an estimated filtration rate of less than 33 $\mathrm{mL} / \mathrm{min}$ gained a survival benefit from HKT over heart transplantation alone.

In our experience with 7 patients who have undergone HKT with single donor allografts all patient are alive at a mean follow-up of 45 months \pm 24 months (range, 75 months - 12 months) which supports the satisfactory results of this procedure.

Some advantages can be noted in using the same donor and a single operative session for both organs. Firstly the recipients have a single exposure to alloantigen avoiding further antigenic stimulation. Secondly, they have a single induction immunosuppressive treatment and, thirdly, when the surgical procedure is carried out in the same surgical session they avoid a subsequent anaesthesia and also the cold renal storage is reduced. Some authors $[6,9]$ have stated a preference for a staged approach involving a later second operative procedure. However, in the experience of kidney transplantation, prolonged cold ischemic time (CIT) is a well-known risk factor for delayed graft function [10] which is itself associated to lower graft function and graft survival rate in the long term [11].

In our experience, except for one staged procedure which was necessary due to instable hemodynamic conditions, all kidneys were immediately implanted after the closure of the chest allowing for a CIT of no longer than 10 hours.

Of note, many studies describe a low rate of rejection in HKT $[3,6,9]$ which is in accordance with another recent analysis from UNOS clinical data [12] that showed that both kidney and heart allograft rejections are reduced when compared with each respective allograft alone ( $17 \%$ vs $24 \%$, $p<0.001$ and $26 \%$ vs $52 \%$, $\mathrm{p}<0.001$; respectively). More generally they found that the heart liver and kidney allografts are themselves protected from rejection and that they protect the other organ when transplanted from the same donor. It appears that any organ transplant combination may induce tolerance or reduce host immunoresponsiveness, but the mechanisms of this immune event in human patients are not clearly understood even if various hypotheses have been suggested [3,13,14].

In our study population, four out of seven patients had no acute rejection episode of either organ and no simultaneous rejection was noted. Despite the limited data we have, this supports the fact that simultaneous rejection is rare in HKT [15] thus suggesting that surveillance of both organs is advisable and could be carried out separately for each transplanted organ.

Despite the good clinical HKT results, the use for two organs for one patient of such a scarcity of donors may represent an ethical dilemma. We believe that this issue can be at least partially settled with an optimal candidate selection that is guided by the indications for heart transplantation, viewing the combined kidney transplant as a possibility to remove a contraindication to heart transplant alone. Of note, as opposed to end-stage kidney disease that can be artificially treated with long-term dialysis, no mechanical device is currently represent a valuable option as a substitute for heart transplantation for resolving end stage heart failure. Thus, heart transplantation may be the only lifesaving option in patients with end stage heart failure that additionally suffer from end-stage kidney disease. On the other hand, we do not believe that HKT should be an option permitting kidney transplant in patients with end-stage kidney disease and severe but nonsymptomatic heart disease, because heart transplant is not proven to improve survival in these patients as compared with optimal medical treatment for heart failure [16]. Our current policy is to consider HKT in patients with an indication for heart transplant, a low comorbidity profile, and a creatinine clearance of less than $30 \mathrm{~mL} / \mathrm{min}$ due to parenchyma kidney disease [17], (i.e. excluding patients with renal insufficiency secondary to low-cardiac output alone).

In conclusion, our results support the previously reported data about HKT, suggesting that this procedure has become a valuable option for selected patients needing a heart transplant with concurrent severe renal failure. Donor scarcity underlines the need for a careful and stringent selection of candidate patients in order to optimize the best outcome for both organs.

\section{References}

[1] J. Norman, D. Cooley, B., Kahan, et al., "Total Support of the Circulation of a Patient with Post-Cardiotomy Stone-Heart Syndrome by a Partial Artificial Heart (ALVAD) for 5 Days Followed by Heart and Kidney Transplantation,” Lancet, Vol. 1, No. 8074 , 1978, pp. 1125-1127. doi:10.1016/S0140-6736(78)90301-X

[2] S. Livesey, K. Rolles, R. Calne, J. Wallwork and T. A. H. English, "Successful Simultaneous Heart and Kidney Transplantation Using the Same Donor," Clinical Transplantation, Vol. 2, 1988, pp. 1-4.

[3] J. Narula, L. E. Bennett, T. DiSalvo, J. D. Hosenpud, M. J. Semigran and G. W. Dec, "Outcomes in Recipients of 
Combined Heart-Kidney Transplantation: Multiorgan, Same-Donor Transplant Study of the International Society of Heart and Lung Transplantation/United Network for Organ Sharing Scientific Registry,” Transplantation, Vol. 63, No. 6, 1997, pp. 861-867. doi:10.1097/00007890-199703270-00012

[4] G. D. Trachiotis, J. D. Vega, T. S. Johnston, et al., “TenYear Follow-up in Patients with Combined Heart and Kkidney Transplantation," Journal of Thoracic and Cardiovascular Surgery, Vol. 126, No. 6, 2003, pp. 20652071. doi:10.1016/j.jtcvs.2003.07.009

[5] S. S. Wang, N. K. Chou, N. H. Chi, et. al., "Simultaneous Heart and Kidney Transplantation for Combined Cardiac and Renal Failure,” Transplantation Proceedings, Vol. 38, No. 7, 2006, pp. 2135-2137. doi:10.1016/j.transproceed.2006.06.002

[6] J. L. Hermsen, D. S. Nath, A. M. del Rio, J. B. Eickstaedt, C. Wigfield, J. D. Lindsey and N. M. Edwards, "Combined Heart-Kidney Transplantation: The University of Wisconsin Experience," Journal of Heart and Lung Transplantation, Vol. 26, No. 11, 2007; pp. 1119-1126. doi:10.1016/j.healun.2007.08.011

[7] G. Bruschi, L. Botta, T. Colombo, et al., "Combined Heart and Kidney Transplantation: Long-Term Analysis of Renal Function and Major Adverse Events at 20 Years,” Transplantation Proceedings, Vol. 42, No. 4, 2010, pp. 1283-1285. doi:10.1016/j.transproceed.2010.03.064

[8] M. J. Russo, A. Rana, J. M. Chen, et al., "Pretransplantation Patient Characteristics and Survival Following Combined Heart and Kidney Transplantation,” Archives of Surgery, Vol. 144, No. 3, 2009, pp. 241-246. doi:10.1001/archsurg.2008.559

[9] C. Blanche, A. Kamlot, D. A. Blanche, B. Kearney, A. V. Wong, L. S. Czer and A. Trento, "Combined Heart-Kidney Transplantation with Single-Donor Allografts,” Journal of Thoracic and Cardiovascular Surgery, Vol. 122, No. 3, 2001, pp. 495-500. doi:10.1067/mtc.2001.115700

[10] W. D. Irish, D. A. McCollum, R. J. Tesi, et al., "Nomo- gram for Predicting the Likelihood of Delayed Graft Function in Adult Cadaveric Renal Transplant Recipients," Journal of the American Society of Nephrology, Vol. 14, No. 11, 2003, pp. 2967-2974. doi:10.1097/01.ASN.0000093254.31868.85

[11] S. G. Yarlagadda, S. G. Coca, R. N. Formica Jr, E. D. Poggio and C. R. Parikh, “Association between Delayed Graft Function and Allograft and Patient Survival: A Systematic Review and Meta-Analysis,” Nephrology Dialysis Transplantation, Vol. 24, No. 3, 2009, pp. 10391047. doi:10.1093/ndt/gfn667

[12] A. Rana, S. Robles, M. J. Russo, et al., "The Combined Organ Effect: Protection against Rejection?” Annals of Surgery, Vol. 248, No. 5, 2008, pp. 871-879. doi:10.1097/SLA.0b013e31817fc2b8

[13] T. E. Starzl, A. J. Demetris, N. Murase, et al., "Chimerism after Organ Transplantation,” Current Opinion in Nephrology and Hypertension, Vol. 6, No. 3, 1997, pp. 292-298. doi:10.1097/00041552-199705000-00015

[14] N. Perico and G. Remuzzi, “Acquired Transplant Tolerance,” International Journal of Clinical and Laboratory Research, Vol. 27, No. 3, 1997, pp. 165-177. doi:10.1007/BF02912453

[15] J. A. Castillo-Lugo and K. R. Brinker, “An Overview of Combined Heart and Kidney Transplantation," Current Opinion in Cardiolog, Vol. 14, No. 2, 1999, pp. 121-125. doi:10.1097/00001573-199903000-00008

[16] N. R. Banner, C. A. Rogers and R. S. Bonser, United Kingdom Cardiothoracic Transplant Audit Steering Group, "Effect of Heart Transplantation on Survival in Ambulatory and Decompensated Heart Dailure," Transplantation, Vol. 86, No. 11, 2008, pp. 1515-1522. doi:10.1097/TP.0b013e31818b3328

[17] G. Mosconi, L. Panicali, E. Persici, et al., "Native Kidney Function after Renal Transplantation Combined with Other Solid Organs in Preemptive Patients,” Transplantation Proceedings, Vol. 42, No. 4, 2010, pp. 1017-1020. doi:10.1016/j.transproceed.2010.03.125 